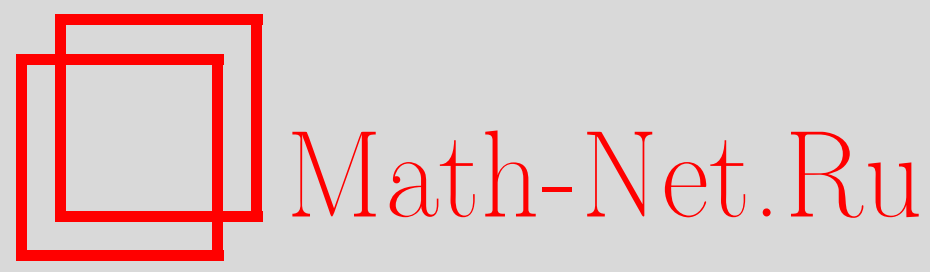

М. К. Потапов, Г. Н. Казимиров, О приближении алгебраическими многочленами функций, имеющих данный порядок $k$-го обобщенного модуля гладкости, Матем. заметки, 1998, том 63, выпуск 3, 425-436

DOI: https://doi.org/10.4213/mzm1299

Использование Общероссийского математического портала Math-Net.Ru подразумевает, что вы прочитали и согласны с пользовательским соглашением http://www.mathnet.ru/rus/agreement

Параметры загрузки:

IP : 54.92 .164 .108

26 апреля 2023 г., 18:17:33

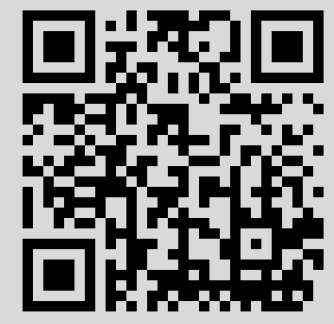




\title{
О ПРИБЛИЖЕНИИ АЛГЕБРАИЧЕСКИМИ МНОГОЧЛЕНАМИ ФУНКЦИЙ, ИМЕЮЩИХ ДАННЫЙ ПОРЯДОК $k$-ГО ОБОБШЕННОГО МОДУЛЯ ГЛАДКОСТИ
}

\author{
М.К. Потапов, Г. Н. Казимиров
}

\begin{abstract}
В работе рассмотрен вопрос о приближении алгебраическими многочленами классов функций, характеризуемых порядком $k$-го обобщенного модуля гладкости, определяемого с помощью оператора обобщенного сдвига Якоби.
\end{abstract}

Библиография: 7 названий.

В данной работе рассматривается вопрос о приближении алгебраическими многочленами классов функций, характеризуемых порядком $k$-го обобщенного модуля гладкости, определяемого с помощью оператора обобщенного сдвига Якоби. Для модулей гладкости первого порядка, определяемых с помошью того же оператора, этот вопрос рассматривался в работе [1].

1. Будем говорить, что $f \in L_{p}$, если для $1 \leqslant p<\infty$ функция $f$ измерима на отрезке $[-1,1]$ и

$$
\|f\|_{p}=\left(\int_{-1}^{1}|f(x)|^{p} d x\right)^{1 / p}<\infty
$$

а для $p=\infty$ функция $f$ непрерьвна на отрезке $[-1,1]$ и

$$
\|f\|_{\infty}=\max _{-1 \leqslant x \leqslant 1}|f(x)| .
$$

Будем также говорить, что $f \in L_{p, \alpha, \beta}, 1 \leqslant p \leqslant \infty$, если $f(x)(1-x)^{\alpha}(1+x)^{\beta} \in L_{p}$, причем

$$
\|f\|_{p, \alpha, \beta}=\left\|f(x)(1-x)^{\alpha}(1+x)^{\beta}\right\|_{p} .
$$

Обозначим через $E_{n}(f)_{p, \alpha, \beta}$ наилучшее приближение функции $f \in L_{p, \alpha, \beta}$ при помощи алгебраических многочленов $P_{n}$ степени не вьше $n-1$ в метрике $L_{p, \alpha, \beta}$, т.е.

$$
E_{n}(f)_{p, \alpha, \beta}=\inf _{P_{n} \in \mathbf{P}}\left\|P_{n}(x)-f(x)\right\|_{p, \alpha, \beta},
$$

где $\mathbf{P}$ - множество алгебраических многочленов степени не выше $n-1(n=1,2, \ldots)$.

Работа выполнена при поддержке Российского фонда фундаментальных исследований, грант № 96-01-00094, и фонда поддержки ведущих научных школ, грант № 96-15-96073. 
Обозначим через $\mathbf{E}(p, \alpha, \beta, \rho)$ класс функций $f \in L_{p, \alpha, \beta}$, удовлетворяющих условию

$$
E_{n}(f)_{p, \alpha, \beta} \leqslant C_{1} n^{-\rho}, \quad \rho>0,
$$

где постоянная $C_{1}$ не зависит от $n(n=1,2, \ldots)$.

Для данных чисел $\nu$ и $\mu(\nu \geqslant \mu \geqslant-1 / 2)$ определим оператор обобщенного сдвига Якоби $T_{t}(f, x, \nu, \mu)$ по правилу:

1) для $\nu=\mu=-1 / 2$

$$
T_{t}(f, x, \nu, \mu)=\frac{1}{2}\left(f\left(x \cos t+\sqrt{1-x^{2}} \sin t\right)+f\left(x \cos t-\sqrt{1-x^{2}} \sin t\right)\right) ;
$$

2) для $\nu=\mu>-1 / 2$

$$
T_{t}(f, x, \nu, \mu)=\frac{1}{\gamma(\nu)} \int_{-1}^{1} f\left(x \cos t+y \sqrt{1-x^{2}} \sin t\right)\left(1-y^{2}\right)^{\nu-1 / 2} d y
$$

3) для $\nu>\mu=-1 / 2$

$T_{t}(f, x, \nu, \mu)=\frac{1}{\gamma(\nu)} \int_{-1}^{1} f\left(x \cos t+y \sqrt{1-x^{2}} \sin t-\left(1-y^{2}\right)(1-x) \sin ^{2} \frac{t}{2}\right)\left(1-y^{2}\right)^{\nu-1 / 2} d y$

4) для $\nu>\mu>-1 / 2$

$$
\begin{gathered}
T_{t}(f, x, \nu, \mu)=\frac{1}{\gamma(\nu, \mu)} \int_{0}^{1} \int_{-1}^{1} f\left(x \cos t+y z \sqrt{1-x^{2}} \sin t-\left(1-y^{2}\right)(1-x) \sin ^{2} \frac{t}{2}\right) \\
\times\left(1-y^{2}\right)^{\nu-\mu-1} y^{2 \mu+1}\left(1-z^{2}\right)^{\mu-1 / 2} d z d y
\end{gathered}
$$

где

$\gamma(\nu)=\int_{-1}^{1}\left(1-y^{2}\right)^{\nu-1 / 2} d y, \quad \gamma(\nu, \mu)=\int_{0}^{1} \int_{-1}^{1}\left(1-y^{2}\right)^{\nu-\mu-1} y^{2 \mu+1}\left(1-z^{2}\right)^{\mu-1 / 2} d z d y$.

Для $f \in L_{p, \alpha, \beta}$ и данных чисел $\nu$ и $\mu(\nu \geqslant \mu \geqslant-1 / 2)$ введем $k$-ю обобщенную разность Якоби по правилу

$$
\begin{gathered}
\Delta_{t}^{1}(f, x, \nu, \mu)=T_{t}(f, x, \nu, \mu)-f(x), \\
\Delta_{t_{1}, \ldots, t_{k}}^{k}(f, x, \nu, \mu)=\Delta_{t_{k}}^{1}\left(\Delta_{t_{1}, \ldots, t_{k-1}}^{k-1}(f, x, \nu, \mu), x, \nu, \mu\right), \quad k=2,3, \ldots,
\end{gathered}
$$

и $k$-й обобщенный модуль гладкости Якоби по формуле

$$
\widetilde{w}_{k}(f, \delta, \nu, \mu)_{p, \alpha, \beta}=\sup _{\substack{\left|t_{i}\right| \leqslant \delta \\ i=1, \ldots, k}}\left\|\Delta_{t_{1}, \ldots, t_{k}}^{k}(f, x, \nu, \mu)\right\|_{p, \alpha, \beta}
$$

Обозначим через $\mathbf{H}(p, \alpha, \beta, \rho, \nu, \mu, r)$ класс функций $f \in L_{p, \alpha, \beta}$, удовлетворяющих условию

$$
\widetilde{w}_{r}(f, \delta, \nu, \mu)_{p, \alpha, \beta} \leqslant C_{1} \delta^{\rho}, \quad \rho>0,
$$

где постоянная $C_{1}$ не зависит от $\delta$. 
Введем также обозначения

$$
\begin{gathered}
D_{x, \nu, \mu}=(1-x)^{-\nu}(1+x)^{-\mu} \frac{d}{d x} \cdot(1-x)^{\nu+1}(1+x)^{\mu+1} \frac{d}{d x}, \\
D_{x, \nu, \mu}^{1}=D_{x, \nu, \mu}, \quad D_{x, \nu, \mu}^{k}=D_{x, \nu, \mu}\left(D_{x, \nu, \mu}^{k-1}\right), \\
T_{t}^{1}(f, x, \nu, \mu)=T_{t}(f, x, \nu, \mu)=T_{t} f, \quad T_{t}^{k}(f, x, \nu, \mu)=T_{t}^{1}\left(T_{t}^{k-1}(f, x, \nu, \mu), x, \nu, \mu\right), \\
T_{t_{1}, \ldots, t_{k}}^{k}(f, x, \nu, \mu)=T_{t_{k}}^{1}\left(T_{t_{1}, \ldots, t_{k-1}}^{k-1}(f, x, \nu, \mu), x, \nu, \mu\right), \\
k=2,3, \ldots .
\end{gathered}
$$

Обозначим через $P_{n}^{(\nu, \mu)}(x)(n=1,2, \ldots)$ алгебраические многочлены степени $n-1$, ортогональные друг другу на отрезке $[-1,1]$ с весом $(1-x)^{\nu}(1+x)^{\mu}$ и нормированные условием $P_{n}^{(\nu, \mu)}(1)=1$.

2. Сформулируем основной результат работы.

ТЕОремА 1. Пусть числа $p, \nu, \mu$ u $r$ таковы, что $1 \leqslant p \leqslant \infty, \nu \geqslant \mu \geqslant-1 / 2$, $r \in \mathbb{N}$. Пусть числа $\alpha$ и $\beta$ удовлетворяют следующим условиям:

\begin{tabular}{|c|c|c|c|}
\hline & $n p u p=1$ & $n p u 1<p<\infty$ & $n p u p=\infty$ \\
\hline$n p u \nu=\mu=-\frac{1}{2}$ & $\alpha=\beta=-\frac{1}{2}$ & $-\frac{1}{2 p} \leqslant \alpha<\frac{1}{2}-\frac{1}{p}$ & $0 \leqslant \alpha<\frac{1}{2}$ \\
\hline$n p u \nu=\mu>-\frac{1}{2}$ & $-\frac{1}{2}<\alpha \leqslant \nu$ & $-\frac{1}{2 p}<\alpha<\nu+1-\frac{1}{p}$ & $0 \leqslant \alpha<\frac{1}{2}$ \\
\hline npu $\nu>\mu=-\frac{1}{2}<\beta \leqslant \nu$ & $-\frac{1}{2 p}<\beta<\nu+1-\frac{1}{p}$ & $0 \leqslant \beta<\nu+1$ \\
\hline npu $\nu>\mu>-\frac{1}{2}$ & $-\frac{1}{2}<\alpha \leqslant \nu$ & $-\frac{1}{2 p}<\alpha<\nu+1-\frac{1}{p}$ & $0 \leqslant \alpha<\nu+1$ \\
& $-\frac{1}{2}<\alpha \leqslant \nu$ & $-\frac{1}{2 p}<\alpha<\nu+1-\frac{1}{p}$ & $0 \leqslant \beta<\frac{1}{2}$ \\
\hline & $-\frac{1}{2}<\beta \leqslant \mu$ & $-\frac{1}{2 p}<\beta<\mu+1-\frac{1}{p}$ & $0 \leqslant \beta<\mu+1$ \\
\hline
\end{tabular}

Пусть число $\rho$ такое, что $2 r>\rho>\rho_{0}, u$

$$
\rho_{0}=\left\{\begin{array}{l}
2 \max \left(|\alpha-\beta|, \min (\alpha, \beta)-\mu+\frac{1}{2 p}-\frac{1}{2},|\alpha-\beta|+\min (\alpha, \beta)-\mu+\frac{1}{2 p}-\frac{1}{2}\right) \\
n p u \nu=\mu \geqslant-\frac{1}{2}, \\
2 \max \left(0, \beta-\alpha,(\alpha-\beta)-(\nu-\mu),\left(\alpha+\frac{1}{2 p}\right)-\left(\nu+\frac{1}{2}\right),\left(\beta+\frac{1}{2 p}\right)-\left(\mu+\frac{1}{2}\right)\right) \\
\quad n p u \nu>\mu \geqslant-\frac{1}{2} .
\end{array}\right.
$$

Тогда класс функиий $\mathbf{H}(p, \alpha, \beta, \rho, \nu, \mu, r)$ совпадает с классом функиий $\mathbf{E}(p, \alpha, \beta, \rho)$. 
ЗАмЕчАниЕ. Теорема 1 содержит в себе прямую и обратную теоремы теории приближений для модулей гладкости $\widetilde{w}_{k}(f, \delta, \nu, \mu)_{p, \alpha, \beta}$ степенного типа. Если ослабить условия теоремы 1 , то для обших модулей гладкости $\widetilde{w}_{k}(f, \delta, \nu, \mu)_{p, \alpha, \beta}$ справедливо следуюшее утверждение.

Теорема 2. Пусть числа $p, \nu, \mu$ u $r$ таковы, что $1 \leqslant p \leqslant \infty, \nu \geqslant \mu \geqslant-1 / 2$, $r \in \mathbb{N}$. Пусть числа $\alpha$ и выбраны по правилу:

1) если $\nu=\mu=-1 / 2$, то $\alpha=\beta=-1 /(2 p)$ nрu $1 \leqslant p \leqslant \infty$;

2) если $\nu=\mu>-1 / 2$, по $\alpha=\beta u-1 / 2<\alpha \leqslant \nu$ nри $p=1,-1 /(2 p)<\alpha<\nu+$ $1 / 2-1 /(2 p)$ npu $1<p<\infty, 0 \leqslant \alpha<\nu+1 / 2$ npu $p=\infty$

3) если $\nu>\mu=-1 / 2$, то $\beta=-1 /(2 p),-1 / 2<\alpha \leqslant \nu$ nри $p=1,-1 /(2 p)<\alpha<$ $\nu+1 / 2-1 /(2 p)$ npu $1<p<\infty, 0 \leqslant \alpha<\nu+1 / 2$ npu $p=\infty$;

4) если $\nu>\mu>-1 / 2$, то $\nu-\mu>\alpha-\beta \geqslant 0 u-1 / 2<\beta<\mu$ nри $p=1$, $\nu-\mu>\alpha-\beta>0 u-1 /(2 p)<\beta<\mu+1 / 2-1 /(2 p)$ npu $1<p<\infty$, $\nu-\mu>\alpha-\beta \geqslant 0 u 0 \leqslant \beta<\mu+1 / 2$ при $p=\infty$.

Тогда для $f \in L_{p, \alpha, \beta}$ справедливы неравенства

$$
C_{1} E_{n}(f)_{p, \alpha, \beta} \leqslant \widetilde{w}_{r}\left(f, \frac{1}{n}, \nu, \mu\right)_{p, \alpha, \beta} \leqslant \frac{C_{2}}{n^{2 r}} \sum_{m=1}^{n} m^{2 r-1} E_{m}(f)_{p, \alpha, \beta},
$$

где положсительные постоянные $C_{1}$ и $C_{2}$ не зависят от $f$ u $n(n=1,2, \ldots)$.

В этой работе будет доказана теорема 1. Теорема 2 доказана в [2].

3. Приведем вспомогательные утверждения, необходимые для доказательства основного утверждения.

Лемма 1. Справедливы следующие свойства оператора обобщенного сдвига Якоби:

1) оператор $T_{t}(f, x, \nu, \mu)$ линеен;

2) $T_{t}(f, \cos \theta, \nu, \mu)=T_{\theta}(f, \cos t, \nu, \mu), t, \theta \in \mathbb{R}$;

3) имеет место теорема умнохсения

$$
T_{t}\left(P_{n}^{(\nu, \mu)}, x, \nu, \mu\right)=P_{n}^{(\nu, \mu)}(\cos t) P_{n}^{(\nu, \mu)}(x)
$$

4) если $\nu \geqslant \mu \geqslant-1 / 2$ и функиия $g(x) T_{t}(f, x, \nu, \mu) \in L_{1, \nu, \mu}$ для любого $t \in \mathbb{R}$, то верно равенство

$$
\int_{-1}^{1} g(x) T_{t}(f, x, \nu, \mu)(1-x)^{\nu}(1+x)^{\mu} d x=\int_{-1}^{1} f(x) T_{t}(g, x, \nu, \mu)(1-x)^{\nu}(1+x)^{\mu} d x .
$$

Свойства 1) и 2) следуют из определения оператора обобщенного сдвига Якоби. Доказательство свойства 3) см. в [3], [4], а свойства 4) - в [5], [1].

Лемма 2 [6]. Пусть $f \in L_{p, \alpha, \beta}, u$ пусть числа $\alpha, \beta, \delta, \sigma u \nu, \mu$ таковы, что $\alpha>-1 / p, \beta>-1 / p$ для $1 \leqslant p<\infty u \alpha \geqslant 0, \beta \geqslant 0$ для $p=\infty ; \delta \geqslant 0, \sigma \geqslant 0$, $\lambda>\lambda_{0}=2 \max (\sigma, \delta)$. 
Если существует последовательность алгебраических многочленов $P_{2^{n}}(x)(n=$ $0,1,2, \ldots)$ степени не выше $2^{n}-1$ таких, что

$$
\left\|\left(f(x)-P_{2^{n}}(x)\right)(1-x)^{\alpha+\sigma}(1+x)^{\beta+\delta}\right\|_{p} \leqslant \frac{C_{1}}{2^{n \lambda}},
$$

то справедливы также неравенства

$$
\left\|\left(f(x)-P_{2^{n}}(x)\right)(1-x)^{\alpha}(1+x)^{\beta}\right\|_{p} \leqslant \frac{C_{2}}{2^{n\left(\lambda-\lambda_{0}\right)}},
$$

әде постоянные $C_{1}$ и $C_{2}$ не зависят от $n(n=0,1,2, \ldots)$.

Лемма 3 [7]. Пусть $P_{n}(x)$ - алгебраический многочлен степени не выше $n-1$, и пусть числа $p, \alpha, \beta, \sigma_{1}$ и $\sigma_{2}$ таковы, что $1 \leqslant p \leqslant \infty, \alpha \geqslant-1 /(2 p), \beta \geqslant-1 /(2 p)$, $\sigma_{1} \geqslant 0, \sigma_{2} \geqslant 0$.

Тогда справедливы неравенства

$$
\begin{gathered}
\left\|P_{n}^{\prime}(x)\right\|_{p, \alpha+1 / 2, \beta+1 / 2} \leqslant C_{1} n\left\|P_{n}(x)\right\|_{p, \alpha, \beta}, \\
\left\|P_{n}(x)\right\|_{p, \alpha, \beta} \leqslant C_{2} n^{2 \max \left(\sigma_{1}, \sigma_{2}\right)}\left\|P_{n}(x)\right\|_{p, \alpha+\sigma_{1}, \beta+\sigma_{2}},
\end{gathered}
$$

где постоянные $C_{1}$ и $C_{2}$ не зависят от $n(n=1,2, \ldots)$.

Лемма 4. а) Пусть даны числа $p, \nu$ и $\mu$ такие, что $1 \leqslant p \leqslant \infty, \nu=\mu \geqslant-1 / 2, u$ пусть числа $\alpha$ и $\beta$ выбраны так, что $\alpha \geqslant-1 /(2 p)$ и $\beta \geqslant-1 /(2 p)$ при $1 \leqslant p<\infty u$ $\mu=-1 / 2, \alpha>-1 /(2 p)$ u $\beta>-1 /(2 p)$ nрu $1 \leqslant p<\infty u \mu>-1 / 2, \alpha \geqslant 0$ u $\beta \geqslant 0$ nри $p=\infty u \mu \geqslant-1 / 2$. Пусть $\sigma_{2}=\min (\alpha, \beta)$,

$$
\sigma= \begin{cases}\sigma_{2}+\frac{1}{2 p}, & \text { если } \mu=-\frac{1}{2} \\ \left(\sigma_{2}+\frac{1}{2 p}\right)-\left(\mu+\frac{1}{2}\right)+a, & \text { еслu } \mu>-\frac{1}{2} u \sigma_{2}+\frac{1}{2 p} \geqslant \mu+\frac{1}{2}, \\ 0, & \text { еслu } \mu>-\frac{1}{2} u \sigma_{2}+\frac{1}{2 p}<\mu+\frac{1}{2},\end{cases}
$$

əде $a=0$ при $p=1$ u $0<a<\mu+1 / 2$ при $1<p \leqslant \infty$.

Тогда

$$
\begin{gathered}
\left\|T_{t} \varphi\right\|_{p, \alpha, \beta} \leqslant C\left(\|\varphi\|_{p, \alpha, \beta}+t^{2|\alpha-\beta|}\|\varphi\|_{p, \sigma_{2}, \sigma_{2}}+t^{2 \sigma}\|\varphi\|_{p, \alpha-\sigma, \beta-\sigma}\right. \\
\left.+t^{2(\sigma+|\alpha-\beta|)}\|\varphi\|_{p, \sigma_{2}-\sigma, \sigma_{2}-\sigma}\right)
\end{gathered}
$$

әде постоянная $C$ не зависит от $t u \varphi$.

б) Пусть даны числа $p, \nu$ и $е$ такие, что $1 \leqslant p \leqslant \infty, \nu>\mu \geqslant-1 / 2$, и пусть числа $\alpha$ и $\beta$ виббраны так, что $\alpha>-1 /(2 p)$ и $\beta \geqslant-1 /(2 p)$ при $1 \leqslant p<\infty u$ $\mu=-1 / 2, \alpha>-1 /(2 p)$ u $\beta>-1 /(2 p)$ nрu $1 \leqslant p<\infty u \mu>-1 / 2, \alpha \geqslant 0$ u $\beta \geqslant 0$ nри $p=\infty u \mu \geqslant-1 / 2$. Пусть

$$
\delta= \begin{cases}0, & \text { если } \beta<\alpha, \\ \beta-\alpha+\varepsilon, & \text { если } \beta \geqslant \alpha,\end{cases}
$$


где $0<\varepsilon<\min (\nu-\mu, \alpha+1 /(2 p), \mu+1 /(2 p))$ npu $1 \leqslant p<\infty u \mu>-1 / 2$, $0<\varepsilon<\min (\nu+1 / 2, \alpha+1 /(2 p)) n p u 1 \leqslant p<\infty u \mu=-1 / 2, \varepsilon=0$ npu $p=\infty$;

$$
\gamma= \begin{cases}(\alpha-\beta)-(\nu-\mu)+b, & \text { если } \alpha-\beta \geqslant \nu-\mu, \\ 0, & \text { если } \alpha-\beta<\nu-\mu\end{cases}
$$

əде $b=0$ при $p=1 u 0<b<\nu-\mu$ nрu $1<p \leqslant \infty$;

$$
\sigma= \begin{cases}\beta+\frac{1}{2 p}, & \text { если } \beta<\alpha u \mu=-\frac{1}{2} \\ \alpha+\frac{1}{2 p}-\varepsilon, & \text { если } \beta \geqslant \alpha u \mu=-\frac{1}{2} \\ \left(\beta+\frac{1}{2 p}\right)-\left(\mu+\frac{1}{2}\right)+a, & \text { если } \beta<\alpha, \mu>-\frac{1}{2} u \beta+\frac{1}{2 p} \geqslant \mu+\frac{1}{2} \\ 0, & \text { если } \beta<\alpha, \mu>-\frac{1}{2} u \beta+\frac{1}{2 p}<\mu+\frac{1}{2} \\ \left(\alpha+\frac{1}{2 p}\right)-\left(\mu+\frac{1}{2}\right)+a, & \text { если } \beta \geqslant \alpha, \mu>-\frac{1}{2} u \alpha+\frac{1}{2 p} \geqslant \mu+\frac{1}{2} \\ 0, & \text { если } \beta \geqslant \alpha, \mu>-\frac{1}{2} u \alpha+\frac{1}{2 p}<\mu+\frac{1}{2}\end{cases}
$$

əде $a=0$ при $p=1 u 0<a<\mu+1 / 2-\varepsilon$ прu $1<p \leqslant \infty$.

Tогда

$$
\begin{aligned}
\left\|T_{t} \varphi\right\|_{p, \alpha, \beta} \leqslant C & \left(\|\varphi\|_{p, \alpha, \beta}+t^{2 \delta}\|\varphi\|_{p, \alpha, \beta-\delta}+t^{2 \gamma}\|\varphi\|_{p, \alpha-\gamma, \beta}\right. \\
& \left.+t^{2 \sigma}\|\varphi\|_{p, \alpha-\sigma, \beta-\sigma}+t^{2(\gamma+\sigma)}\|\varphi\|_{p, \alpha-\delta, \beta-\delta-\sigma}\right)
\end{aligned}
$$

әде постоянная $C$ не зависит от $t u \varphi$.

Лемма 4 доказывается заменами переменных.

Лемма 5. Пусть даны числа $p, \nu$ и $\mu$ такие, что $1 \leqslant p \leqslant \infty, \nu \geqslant \mu \geqslant-1 / 2$. Пусть числа $\alpha$ и выбраны так, что $\alpha \leqslant \nu$ в $\beta \leqslant \mu$ при $p=1, \alpha<\nu+1-1 / p u$ $\beta<\mu+1-1 / p$ npu $1<p \leqslant \infty$.

Тогда если $f \in L_{p, \alpha, \beta}$, mо $f \in L_{1, \nu, \mu} u\|f\|_{1, \nu, \mu} \leqslant C\|f\|_{p, \alpha, \beta}$, где постоянная $C$ не зависит от $f$.

Лемма 5 доказьвается простьми оценками.

4. Построим алгебраический многочлен, с помощью которого будет осуществляться нужное приближение.

ТЕОремА 3. Если $f \in L_{1, \nu, \mu}, \nu \geqslant \mu \geqslant-1 / 2, q \in \mathbb{N}$, то функиия

$$
\begin{gathered}
Q(x)=\int_{0}^{\pi} \cdots \int_{0}^{\pi}\left(\Delta_{t_{1}, \ldots, t_{r}}^{r}(f, x, \nu, \mu)-(-1)^{r} f(x)\right) \prod_{s=1}^{r} \sin ^{2 \nu+1} \frac{t_{s}}{2} \cdot \cos ^{2 \mu+1} \frac{t_{s}}{2} \\
\times\left(\frac{\sin \left(m t_{s} / 2\right)}{\sin \left(t_{s} / 2\right)}\right)^{2 q+4} d t_{1} \cdots d t_{r}
\end{gathered}
$$

есть алгебраический многочлен степени не выше $(q+2)(m-1)$. 
ДокАЗАТЕЛЬСТво. Для доказательства теоремы 3 достаточно доказать, что для любого $k=1,2, \ldots, r$

$$
\begin{gathered}
Q_{1}(x)=\int_{0}^{\pi} \cdots \int_{0}^{\pi} T_{t_{1}, \ldots, t_{k}}^{k}(f, x, \nu, \mu) \prod_{s=1}^{r} \sin ^{2 \nu+1} \frac{t_{s}}{2} \cdot \cos ^{2 \mu+1} \frac{t_{s}}{2} \\
\times\left(\frac{\sin \left(m t_{s} / 2\right)}{\sin \left(t_{s} / 2\right)}\right)^{2 q+4} d t_{1} \cdots d t_{r}
\end{gathered}
$$

есть алгебраический многочлен степени не вьше $(q+2)(m-1)$.

Делая замену переменных $x=\cos \theta$ и пользуясь свойством 2$)$ оператора обобщенного сдвига Якоби (см. лемму 1), получим

$$
\begin{gathered}
Q_{1}(\cos \theta)=\int_{0}^{\pi} \cdots \int_{0}^{\pi} T_{\theta}\left(T_{t_{1}, \ldots, t_{k-1}}^{k-1} f, \cos t_{k}, \nu, \mu\right) \prod_{s=1}^{r} \sin ^{2 \nu+1} \frac{t_{s}}{2} \cdot \cos ^{2 \mu+1} \frac{t_{s}}{2} \\
\times\left(\frac{\sin \left(m t_{s} / 2\right)}{\sin \left(t_{s} / 2\right)}\right)^{2 q+4} d t_{1} \cdots d t_{r} .
\end{gathered}
$$

В силу того, что функция $(\sin (m t / 2) / \sin (t / 2))^{2 q+4}$ есть четньй тригонометрический полином порядка $(q+2)(m-1)$ и любой алгебраический многочлен можно представить в виде линейной комбинации многочленов $P_{n}^{(\nu, \mu)}(x)$, имеем

$$
\begin{aligned}
\left(\frac{\sin (m t / 2)}{\sin (t / 2)}\right)^{2 q+4} & =\sum_{k=0}^{(q+2)(m-1)} a_{k} \cos k t=\sum_{k=0}^{(q+2)(m-1)} b_{k} \cos ^{k} t \\
& =\sum_{i=1}^{(q+2)(m-1)+1} c_{i} P_{i}^{(\nu, \mu)}(\cos t) .
\end{aligned}
$$

Используя это представление, можем записать, что

$$
\begin{aligned}
Q_{1}(\cos \theta)= & \sum_{i=1}^{(q+2)(m-1)+1} c_{i} \int_{0}^{\pi} \cdots \int_{0}^{\pi} T_{\theta}\left(T_{t_{1}, \ldots, t_{k-1}}^{k-1} f, \cos t_{k}, \nu, \mu\right) \\
& \times P_{i}^{(\nu, \mu)}\left(\cos t_{k}\right) \sin ^{2 \nu+1} \frac{t_{k}}{2} \cdot \cos ^{2 \mu+1} \frac{t_{k}}{2} d t_{k} \\
& \times \prod_{\substack{s=1 \\
s \neq k}}^{r} \sin ^{2 \nu+1} \frac{t_{s}}{2} \cdot \cos ^{2 \mu+1} \frac{t_{s}}{2}\left(\frac{\sin \left(m t_{s} / 2\right)}{\sin \left(t_{s} / 2\right)}\right)^{2 q+4} d t_{1} \cdots d t_{k-1} d t_{k+1} \cdots d t_{r} .
\end{aligned}
$$

Пусть

$$
J_{1}=2^{\nu+\mu+1} \int_{0}^{\pi} T_{\theta}\left(T_{t_{1}, \ldots, t_{k-1}}^{k-1} f, \cos t_{k}, \nu, \mu\right) P_{i}^{(\nu, \mu)}\left(\cos t_{k}\right) \sin ^{2 \nu+1} \frac{t_{k}}{2} \cdot \cos ^{2 \mu+1} \frac{t_{k}}{2} d t_{k} .
$$


Сделаем замену переменной $\cos t_{k}=y$. Тогда в силу свойств 4) и 3 ) оператора обобщенного сдвига Якоби (см. лемму 1) имеем

$$
\begin{aligned}
J_{1} & =\int_{-1}^{1} T_{\theta}\left(T_{t_{1}, \ldots, t_{k-1}}^{k-1} f, y, \nu, \mu\right) P_{i}^{(\nu, \mu)}(y)(1-y)^{\nu}(1+y)^{\mu} d y \\
& =\int_{-1}^{1} T_{t_{1}, \ldots, t_{k-1}}^{k-1}(f, y, \nu, \mu) T_{\theta}\left(P_{i}^{(\nu, \mu)}, y, \nu, \mu\right)(1-y)^{\nu}(1+y)^{\mu} d y \\
& =P_{i}^{(\nu, \mu)}(\cos \theta) \int_{-1}^{1} T_{t_{1}, \ldots, t_{k-1}}^{k-1}(f, y, \nu, \mu) P_{i}^{(\nu, \mu)}(y)(1-y)^{\nu}(1+y)^{\mu} d y .
\end{aligned}
$$

Применяя свойство 3) оператора обобщенного сдвига $k-1$ раз, получим, что

$$
\begin{aligned}
J_{1} & =P_{i}^{(\nu, \mu)}(\cos \theta) \prod_{m=1}^{k-1} P_{i}^{(\nu, \mu)}\left(\cos t_{m}\right) \int_{-1}^{1} f(y) P_{i}^{(\nu, \mu)}(y)(1-y)^{\nu}(1+y)^{\mu} d y \\
& =P_{i}^{(\nu, \mu)}(\cos \theta) \prod_{m=1}^{k-1} P_{i}^{(\nu, \mu)}\left(\cos t_{m}\right) d_{i}
\end{aligned}
$$

где

$$
d_{i}=\int_{-1}^{1} f(y) P_{i}^{(\nu, \mu)}(y)(1-y)^{\nu}(1+y)^{\mu} d y .
$$

Подставляя $J_{1}$ в выражение для $Q_{1}(\cos \theta)$ и полагая

$$
\begin{aligned}
\lambda_{\nu}=2^{-\nu-\mu-1} \int_{0}^{\pi} \cdots \int_{0}^{\pi} \prod_{m=1}^{k-1} & P_{i}^{(\nu, \mu)}\left(\cos t_{m}\right) \prod_{\substack{s=1 \\
s \neq k}}^{r} \sin ^{2 \nu+1} \frac{t_{s}}{2} \cdot \cos ^{2 \mu+1} \frac{t_{s}}{2} \\
& \times\left(\frac{\sin \left(m t_{s} / 2\right)}{\sin \left(t_{s} / 2\right)}\right)^{2 q+4} d t_{1} \cdots d t_{k-1} d t_{k+1} \cdots d t_{r}
\end{aligned}
$$

имеем

$$
Q_{1}(\cos \theta)=\sum_{i=1}^{(q+2)(m-1)+1} c_{i} d_{i} \lambda_{i} P_{i}^{(\mu, \nu)}(\cos \theta) .
$$

Отсюда следует утверждение теоремы 3.

5. ДоказАтЕльСтво теоремы 1. Докажем сначалавложение $\mathbf{H}(p, \alpha, \beta, \rho, \nu, \mu, r) \subset$ $\mathbf{E}(p, \alpha, \beta, \rho)$.

Пусть $f \in \mathbf{H}(p, \alpha, \beta, \rho, \nu, \mu, r)$. В силу леммы 5 при условиях теоремы $1 f \in L_{1, \nu, \mu}$. Согласно теореме 3

$$
\begin{gathered}
Q(x)=\frac{1}{\gamma_{m}^{r}} \int_{0}^{\pi} \cdots \int_{0}^{\pi}\left(\Delta_{t_{1}, \ldots, t_{r}}^{r}(f, x, \nu, \mu)-(-1)^{r} f(x)\right) \prod_{s=1}^{r} \sin ^{2 \nu+1} \frac{t_{s}}{2} \cdot \cos ^{2 \mu+1} \frac{t_{s}}{2} \\
\times\left(\frac{\sin \left(m t_{s} / 2\right)}{\sin \left(t_{s} / 2\right)}\right)^{2 q+4} d t_{1} \cdots d t_{r}
\end{gathered}
$$

где

$$
\gamma_{m}=\int_{0}^{\pi} \sin ^{2 \nu+1} \frac{t}{2} \cdot \cos ^{2 \mu+1} \frac{t}{2}\left(\frac{\sin (m t / 2)}{\sin (t / 2)}\right)^{2 q+4} d t
$$


есть алгебраический многочлен степени не вьше $(q+2)(m-1)$.

Для каждого натурального $n$ выберем натуральное число $m$ так, чтобы было вьполнено условие

$$
\frac{n-1}{q+2}<m \leqslant \frac{n-1}{q+2}+1
$$

Тогда алгебраический многочлен $Q(x)$ имеет степень не выше $n-1$.

Рассмотрим $J=E_{n}(f)_{p, \alpha, \beta}$. Ясно, что

$$
\begin{aligned}
J \leqslant & \left\|f(x)-Q(x)(-1)^{r+1}\right\|_{p, \alpha, \beta} \\
= & \frac{1}{\gamma_{m}^{r}} \| \int_{0}^{\pi} \cdots \int_{0}^{\pi} \Delta_{t_{1}, \ldots, t_{r}}^{r}(f, x, \nu, \mu) \prod_{s=1}^{r} \sin ^{2 \nu+1} \frac{t_{s}}{2} \cdot \cos ^{2 \mu+1} \frac{t_{s}}{2} \\
& \times\left(\frac{\sin \left(m t_{s} / 2\right)}{\sin \left(t_{s} / 2\right)}\right)^{2 q+4} d t_{1} \cdots d t_{r} \|_{p, \alpha, \beta} .
\end{aligned}
$$

Применяя обобщенное неравенство Минковского, получим, что

$$
\begin{aligned}
J \leqslant & \frac{1}{\gamma_{m}^{r}} \int_{0}^{\pi} \cdots \int_{0}^{\pi} \sup _{\substack{0 \leqslant u_{i} \leqslant t_{1}+\cdots+t_{r} \\
i=1, \ldots, r}}\left\|\Delta_{u_{1}, \ldots, u_{r}}^{r}(f, x, \nu, \mu)\right\|_{p, \alpha, \beta} \prod_{s=1}^{r} \sin ^{2 \nu+1} \frac{t_{s}}{2} \cdot \cos ^{2 \mu+1} \frac{t_{s}}{2} \\
& \times\left(\frac{\sin \left(m t_{s} / 2\right)}{\sin \left(t_{s} / 2\right)}\right)^{2 q+4} d t_{1} \cdots d t_{r} \\
= & \frac{1}{\gamma_{m}^{r}} \int_{0}^{\pi} \cdots \int_{0}^{\pi} \widetilde{w}_{r}\left(f, t_{1}+\cdots+t_{r}, \nu, \mu\right)_{p, \alpha, \beta} \prod_{s=1}^{r} \sin ^{2 \nu+1} \frac{t_{s}}{2} \cdot \cos ^{2 \mu+1} \frac{t_{s}}{2} \\
& \times\left(\frac{\sin \left(m t_{s} / 2\right)}{\sin \left(t_{s} / 2\right)}\right)^{2 q+4} d t_{1} \cdots d t_{r} .
\end{aligned}
$$

Поскольку $f \in \mathbf{H}(p, \alpha, \beta, \rho, \nu, \mu, r)$, имеем

$$
\begin{aligned}
J \leqslant & \frac{C_{2}}{\gamma_{m}^{r}} \int_{0}^{\pi} \cdots \int_{0}^{\pi}\left(t_{1}+\cdots+t_{r}\right)^{\rho} \prod_{s=1}^{r} \sin ^{2 \nu+1} \frac{t_{s}}{2} \cdot \cos ^{2 \mu+1} \frac{t_{s}}{2} \\
& \times\left(\frac{\sin \left(m t_{s} / 2\right)}{\sin \left(t_{s} / 2\right)}\right)^{2 q+4} d t_{1} \cdots d t_{r} \\
\leqslant & \frac{C_{3}}{\gamma_{m}^{r}} \sum_{j=1}^{r} \int_{0}^{\pi} \cdots \int_{0}^{\pi} t_{j}^{\rho} \prod_{s=1}^{r} \sin ^{2 \nu+1} \frac{t_{s}}{2} \cdot \cos ^{2 \mu+1} \frac{t_{s}}{2}\left(\frac{\sin \left(m t_{s} / 2\right)}{\sin \left(t_{s} / 2\right)}\right)^{2 q+4} d t_{1} \cdots d t_{r} .
\end{aligned}
$$

Из стандартных оценок ядра Джексона вытекает неравенство

$$
\frac{1}{\gamma_{m}} \int_{0}^{\pi} h^{\rho} \cdot \sin ^{2 \nu+1} \frac{h}{2} \cdot \cos ^{2 \mu+1} \frac{h}{2}\left(\frac{\sin (m h / 2)}{\sin (h / 2)}\right)^{2 q+4} d h \leqslant \frac{C_{4}}{m^{\rho}}
$$

где постоянная $C_{4}$ не зависит от $m$.

Используя эту оценку, определение $\gamma_{m}$ и неравенство (1), получим, что $J \leqslant C_{5} n^{-\rho}$, где положительная постоянная $C_{5}$ не зависит от $n(n=1,2, \ldots)$. 
Таким образом, показано, что $f \in E(p, \alpha, \beta, \rho)$. Тем самым, доказано вложение $\mathbf{H}(p, \alpha, \beta, \rho, \nu, \mu, r) \subset \mathbf{E}(p, \alpha, \beta, \rho)$.

Докажем обратное вложение. Пусть $f \in E(p, \alpha, \beta, \rho)$. Тогда найдутся алгебраические многочлены $P_{n}(x)$ степени не вьше $n-1$ такие, что

$$
\left\|f(x)-P_{n}(x)\right\|_{p, \alpha, \beta} \leqslant C_{1} n^{-\rho} .
$$

Образуем многочлены $Q_{k}(x)$ по правилу

$$
Q_{0}(x)=P_{1}(x), \quad Q_{k}(x)=P_{2^{k}}(x)-P_{2^{k-1}}(x), \quad k=1,2, \ldots
$$

Рассмотрим $J=\left\|\Delta_{h_{1}, \ldots, h_{r}}^{r}(f, x, \nu, \mu)\right\|_{p, \alpha, \beta}$.

В силу свойства 1) оператора обобщенного сдвига Якоби для любого целого неотрицательного $N$ имеем

$$
J \leqslant\left\|\Delta_{h_{1}, \ldots, h_{r}}^{r}\left(f-P_{2^{N}}, x, \nu, \mu\right)\right\|_{p, \alpha, \beta}+\left\|\Delta_{h_{1}, \ldots, h_{r}}^{r}\left(P_{2^{N}}, x, \nu, \mu\right)\right\|_{p, \alpha, \beta} .
$$

Так как

$$
P_{2^{N}}(x)=\sum_{k=0}^{N} Q_{k}(x)
$$

имеем

$$
J \leqslant\left\|\Delta_{h_{1}, \ldots, h_{r}}^{r}\left(f-P_{2^{N}}, x, \nu, \mu\right)\right\|_{p, \alpha, \beta}+\sum_{k=0}^{N}\left\|\Delta_{h_{1}, \ldots, h_{r}}^{r}\left(Q_{k}, x, \nu, \mu\right)\right\|_{p, \alpha, \beta}=A+\sum_{k=1}^{N} J_{k} .
$$

Пусть для каждого $\delta \in(0, \pi)$ выбрано $N$ так, что

$$
\frac{\pi}{2^{N+1}}<\delta \leqslant \frac{\pi}{2^{N}}
$$

Покажем, что для любых $h_{i}(i=1, \ldots, r)$ таких, что $0<h_{i} \leqslant \delta, r \in \mathbb{N}, \rho_{0}<\rho<2 r$, справедливы неравенства

$$
\begin{gathered}
A \leqslant C_{6} \delta^{\rho}, \\
J_{k} \leqslant C_{7} \delta^{2 r} 2^{k(2 r-\rho)} .
\end{gathered}
$$

Пусть $r=1$ и $\varphi(x)=f(x)-P_{2^{N}}(x)$. Тогда

$$
A=\left\|T_{h_{1}}(\varphi, x, \nu, \mu)-\varphi(x)\right\|_{p, \alpha, \beta} \leqslant\left\|T_{h_{1}}(\varphi, x, \nu, \mu)\right\|_{p, \alpha, \beta}+\|\varphi(x)\|_{p, \alpha, \beta} .
$$

Применяя лемму 4 , неравенство (2) и лемму 2 , получим, что при $\nu=\mu \geqslant-1 / 2$ и $\rho>2(\sigma+|\alpha-\beta|)$

$$
\begin{aligned}
A \leqslant & C_{8}\left(2^{-N \rho}+h_{1}^{2|\alpha-\beta|} 2^{-N(\rho-2|\alpha-\beta|)}+h_{1}^{2 \sigma} 2^{-N(\rho-2 \sigma)}\right. \\
& \left.+h_{1}^{2(\sigma+|\alpha-\beta|)} 2^{-N(\rho-2(\sigma+|\alpha-\beta|))}\right)
\end{aligned}
$$


а при $\nu>\mu \geqslant-1 / 2$ и $\rho>2 \max (\gamma+\sigma, \delta+\sigma)$

$$
\begin{aligned}
A \leqslant & C_{9}\left(2^{-N \rho}+h_{1}^{2 \delta} 2^{-N(\rho-2 \delta)}+h_{1}^{2 \gamma} 2^{-N(\rho-2 \gamma)}+h_{1}^{2 \sigma} 2^{-N(\rho-2 \sigma)}\right. \\
& \left.+h_{1}^{2(\gamma+\sigma)} 2^{-N(\rho-2(\gamma+\sigma))}+h_{1}^{2(\delta+\sigma)} 2^{-N(\rho-2(\delta+\sigma))}\right)
\end{aligned}
$$

где $\sigma, \sigma_{2}, \delta, \gamma$ выбраны согласно лемме 4.

Поскольку при $\nu=\mu \geqslant-1 / 2$ вьполнено $2(\sigma+|\alpha-\beta|) \leqslant \rho_{0}+d$, где $d$ - любое число из промежутка $0<d<\mu+1 / 2$, а при $\nu>\mu \geqslant-1 / 2$ вьполнено $2 \max (\gamma+\sigma, \delta+\sigma) \leqslant \rho_{0}+d$, где $d$ - любое число из промежутка $0<d<\min (\nu-\mu, \alpha+1 /(2 p), \mu+1 / 2-\varepsilon / 2)$ ( $\varepsilon$ выбрано согласно лемме 4$)$, с учетом $h_{1} \leqslant \delta, 1 / 2^{N}<2 \delta / \pi$ получаем, что $A \leqslant C_{10} \delta^{\rho}$ при $\rho>\rho_{0}+d$. Так как $d$ - любое число из указанного промежутка, то неравенство $A \leqslant C_{10} \delta^{\rho}$ справедливо при любом $\rho>\rho_{0}$.

Итак, неравенство (4) для $r=1$ доказано.

Докажем для $r=1$ неравенство (5). Поскольку любой многочлен можно представить в виде линейной комбинации многочленов Якоби, в силу свойства 3) оператора обобщенного сдвига Якоби

$$
T_{h} Q_{k}(x)=\sum_{s=1}^{2^{k}} c_{s} P_{s}^{(\nu, \mu)}(\cos h) P_{s}^{(\nu, \mu)}(x),
$$

где $T_{h} f=T_{h}(f, x, \nu, \mu)$.

Используя равенства (6) и $D_{x, \nu, \mu} P_{s}^{(\nu, \mu)}(x)=s(s+\nu+\mu+1) P_{s}^{(\nu, \mu)}(x)$, легко проверить, что

$$
T_{h} D_{x, \nu, \mu} Q_{k}(x)=D_{\cos h, \nu, \mu} T_{h} Q_{k}(x) .
$$

Применяя равенство (7), имеем

$$
\begin{aligned}
& \int_{0}^{t} \sin ^{-2 \nu-1} \frac{\omega}{2} \cdot \cos ^{-2 \mu-1} \frac{\omega}{2} \int_{0}^{\omega} \sin ^{2 \nu-1} \frac{u}{2} \cdot \cos ^{2 \mu-1} \frac{u}{2} \cdot T_{u}\left(D_{x, \nu, \mu} Q_{k}(x), x, \nu, \mu\right) d u d \omega \\
& \quad=T_{t}\left(Q_{k}, x, \nu, \mu\right)-Q_{k}(x)
\end{aligned}
$$

Отсюда следует, что

$$
\begin{aligned}
J_{k}= & \left\|T_{h_{1}}\left(Q_{k}, x, \nu, \mu\right)-Q_{k}(x)\right\|_{p, \alpha, \beta} \\
= & \| \int_{0}^{h_{1}} \sin ^{-2 \nu-1} \frac{\omega}{2} \cdot \cos ^{-2 \mu-1} \frac{\omega}{2} \\
& \times \int_{0}^{\omega} \sin ^{2 \nu-1} \frac{u}{2} \cdot \cos ^{2 \mu-1} \frac{u}{2} \cdot T_{u}\left(D_{x, \nu, \mu} Q_{k}(x), x, \nu, \mu\right) d u d \omega \|_{p, \alpha, \beta} .
\end{aligned}
$$

Применяя обобщенное неравенство Минковского, при $0<h_{1} \leqslant \pi / 2$ имеем

$$
J_{k} \leqslant C_{11} h_{1}^{2} \sup _{0 \leqslant u_{1} \leqslant h_{1}}\left\|T_{u_{1}}\left(D_{x, \nu, \mu} Q_{k}(x), x, \nu, \mu\right)\right\|_{p, \alpha, \beta} .
$$

Если $\pi / 2<h_{1}<\pi$, то

$$
J_{k} \leqslant\left\|T_{h_{1}}\left(Q_{k}, x, \nu, \mu\right)-T_{\pi / 2} Q_{k}(x)\right\|_{p, \alpha, \beta}+\left\|T_{\pi / 2}\left(Q_{k}, x, \nu, \mu\right)-Q_{k}(x)\right\|_{p, \alpha, \beta} .
$$


С помощью равенства (7) легко получаем, что

$$
\begin{aligned}
& -\int_{\pi / 2}^{h_{1}} \sin ^{-2 \nu-1} \frac{\omega}{2} \cdot \cos ^{-2 \mu-1} \frac{\omega}{2} \int_{0}^{\omega} \sin ^{2 \nu-1} \frac{u}{2} \cdot \cos ^{2 \mu-1} \frac{u}{2} \\
& \quad \times T_{u}\left(D_{x, \nu, \mu} Q_{k}(x), x, \nu, \mu\right) d u d \omega=T_{h_{1}}\left(Q_{k}, x, \nu, \mu\right)-T_{\pi / 2} Q_{k}(x) .
\end{aligned}
$$

Используя это равенство и обобщенное неравенство Минковского, получим, что в случае $\pi / 2<h_{1} \leqslant \pi$ также выполнено

$$
J_{k} \leqslant C_{12} h_{1}^{2} \sup _{0 \leqslant u_{1} \leqslant h_{1}}\left\|T_{u_{1}}\left(D_{x, \nu, \mu} Q_{k}(x), x, \nu, \mu\right)\right\|_{p, \alpha, \beta}
$$

Таким образом, доказано, что для любого $0<h_{1} \leqslant \pi$

$$
J_{k} \leqslant C_{13} h_{1}^{2} \sup _{0 \leqslant u_{1} \leqslant h_{1}}\left\|T_{u_{1}}\left(D_{x, \nu, \mu} Q_{k}(x), x, \nu, \mu\right)\right\|_{p, \alpha, \beta}
$$

Далее, применяя леммы 4,3 , неравенство (2) и лемму 2 и учитьвая, что $u_{1} \leqslant h_{1} \leqslant \delta \leqslant$ $\pi / 2^{N} \leqslant C_{14} / 2^{k}$ для $k=0,1, \ldots, N$, при $\rho>\rho_{0}$ и $k=0,1, \ldots, N$ имеем

$$
J_{k} \leqslant C_{15} \delta^{2} 2^{k(2-\rho)} .
$$

Итак, при $r=1$ неравенства (4) и (5) доказаны.

Для произвольного $r$ неравенства (4) и (5) доказываются по индукции.

Из неравенства (5) следует, что

$$
\sum_{k=1}^{N} J_{k} \leqslant C_{16} \sum_{k=1}^{N} \delta^{2 r} 2^{k(2 r-\rho)} \leqslant C_{17} \delta^{2 r} 2^{N(2 r-\rho)} .
$$

В силу (3) при $\rho_{0}<\rho<2 r$ имеем

$$
\sum_{k=1}^{N} J_{k} \leqslant C_{18} \delta^{\rho}
$$

Из неравенств (4) и (8) следует, что при $\rho_{0}<\rho<2 r$ выполнено

$$
\widetilde{w}_{r}(f, \delta, \nu, \mu)_{p, \alpha, \beta} \leqslant C_{19} \delta^{\rho} .
$$

Тем самым, доказано вложение $\mathbf{E}(p, \alpha, \beta, \rho) \subset \mathbf{H}(p, \alpha, \beta, \rho, \nu, \mu, r)$.

Теорема 1 полностью доказана.

\section{СПИСОК ЦИТИРОВАННОЙ ЛИТЕРАТУРЫ}

[1] Потапов М.К. Об условиях совпадения некоторых классов функций // Тр. семин. им. И.Г. Петровского. 1981. Т. 6. № 223-238.

[2] Казимиров Г. Н. О теоремах Джексона для $k$-го обобщенного модуля гладкости // Деп. ВИНИТИ 27.12.94. М.: ВИНИТИ 1994. № 3054-В94.

[3] Виленкин Н.Я. Специальные функции и теория представлений групп. М.: Наука, 1965.

[4] Koornwinder T. H. The addition formula for Jacobi polynomials. I: Summary of results // Indag. Math. (N.S.). 1972. V. 75. P. 188-191.

[5] Потапов М. К. О структурных характеристиках классов функций с данным порядком наилучшего приближения // Тр. МИАН. 1975. Т. 134. С. 260-277.

[6] Потапов М.K. О структурных и конструктивных характеристиках некоторых классов функций // Тр. МИАН. 1974. Т. 131. С. 211-231.

[7] Халилова Б. А. О некоторых оценках для полиномов // Изв. АН АзССР. Сер. физ.-матем. 1974. № 2. C. 46-55. 